\title{
A HYDRODYNAMICAL HOMOTOPY CO-MOMENTUM MAP AND A MULTISYMPLECTIC INTERPRETATION OF HIGHER-ORDER LINKING NUMBERS
}

\author{
ANTONIO MICHELE MITI (D) and MAURO SPERA (D)
}

(Received 20 December 2019; accepted 17 December 2020; first published online 11 February 2021)

\author{
Communicated by Robert Yuncken
}

\begin{abstract}
In this paper a homotopy co-momentum map (à la Callies, Frégier, Rogers and Zambon) transgressing to the standard hydrodynamical co-momentum map of Arnol'd, Marsden, Weinstein and others is constructed and then generalized to a special class of Riemannian manifolds. Also, a covariant phase space interpretation of the coadjoint orbits associated to the Euler evolution for perfect fluids, and in particular of Brylinski's manifold of smooth oriented knots, is discussed. As an application of the above homotopy co-momentum map, a reinterpretation of the (Massey) higher-order linking numbers in terms of conserved quantities within the multisymplectic framework is provided and knot-theoretic analogues of first integrals in involution are determined.
\end{abstract}

2020 Mathematics subject classification: primary 53D20; secondary 37J39, 53D99, 57K10, 55S30.

Keywords and phrases: higher-order linking numbers, homotopy co-momentum maps, hydrodynamics, symplectic and multisymplectic geometry.

\section{Introduction}

In this paper we discuss some applications of multisymplectic techniques in a hydrodynamical context. The possibility of applying symplectic techniques therein ultimately comes from Arnol'd's pioneering work culminating in the geometrization of fluid mechanics [1-3, 32]. In particular, in this connection we may mention the paper [40], with its symplectic reinterpretation [36-38], and the general portrait depicted in [6]. Here we wish to apply some recently emerged concepts in multisymplectic geometry (mostly building on [7, 45, 46]) and construct an explicit homotopy co-momentum map [7] in a hydrodynamical setting, leading to a multisymplectic interpretation of the so-called higher-order linking numbers, viewed à la Massey [23, 39, 48]. The construction is generalized to cover connected compact oriented Riemannian

(C) Australian Mathematical Publishing Association Inc. 2021. This is an Open Access article, distributed under the terms of the Creative Commons Attribution licence (http://creativecommons.org/ licenses/by/4.0/), which permits unrestricted re-use, distribution, and reproduction in any medium, provided the original work is properly cited. 
manifolds having vanishing intermediate de Rham groups. Moreover, a covariant phase space interpretation of the multisymplectic setting is outlined.

We make clear from the outset that our constructions, together with the covariant phase space portrait, do not adhere to the standard multisymplectic approach to continuum mechanics set forth, for example, in [20,33] but they are based instead on the peculiar structure of an ideal fluid, whose configuration space is the 'Lie group' of diffeomorphisms preserving a volume form, and the latter is directly taken as a multisymplectic form [8].

The layout of the paper is as follows. First, in Section 2, we give an example of homotopy co-momentum map in fluid mechanics-in the sense of Callies et al. [7] - transgressing to Brylinski's symplectic structure on loop spaces and descending, in turn, to the manifold of smooth oriented knots; see [4, 6] and below for precise definitions. We briefly discuss the (non)equivariance of the above construction with respect to the group of volume-preserving diffeomorphisms of 3-space and we outline a generalization thereof in a Riemannian framework, signalling potential topological obstructions. Moreover, covariant phase space aspects are analysed. In Section 3 we prepare the ground for later applications by depicting a hydrodynamical multisymplectic portrait of basic knot-theoretic objects, used, in Section 4, to reinterpret the Massey higher-order linking numbers in multisymplectic terms: the 1-forms appearing in the hierarchical Massey construction (viewed, in turn, differential-geometrically à la Chen) provide an example of first integrals in involution in a multisymplectic framework. The last section is devoted to gathering together the conclusions and to pointing out possible directions for further research. Appropriate background material is provided within the various sections in order to ease readability.

This paper is an improved version of part of the preprint [34].

\section{Multisymplectic geometry and hydrodynamics of perfect fluids}

In the present section we freely use basic material on symplectic and multisymplectic geometry tailored to our subsequent needs, prominently referring, for additional details, to [37, 48, 49] for the former and to [45, 46] for the latter. For general background on symplectic geometry and (co-)momentum maps we quote, among others $[1,3,21]$.

2.1. Tools in multisymplectic geometry. All our objects are smooth, unless differently specified. A (finite-dimensional) multisymplectic manifold $(M, \omega)$ is a manifold (connected, for simplicity) equipped with a closed $(n+1)$-form $\omega$ (called a multisymplectic form or $n$-plectic form) such that the map $\alpha$, given by

$$
\mathfrak{X}(M) \ni \xi \mapsto \alpha(\xi):=\iota_{\xi} \omega \in \Lambda^{n}(M),
$$

which sends vector fields to $n$-forms (via contraction), is injective [8]. Dropping the last condition leads to the concept of pre-n-plectic form. The $n=1$ case retrieves (pre)symplectic manifolds. 
In the multisymplectic context, the generalization of the (co-)momentum maps of the symplectic case leads to the more refined concept of homotopy co-momentum map, presently to be succinctly reviewed. One first introduces the so-called Roger's Lie n-algebra of observables $L_{\infty}(M, \omega)$ [42]. Referring to [7, 16, 42, 45, 46] for a full coverage of the relevant apparatus, not needed to its full extent here, we just point out that the latter is a graded vector space $L$ whose degree $i$ pieces read

$$
\Lambda_{\text {Ham }}^{n-1}(M), i=0, \quad \Lambda^{n-1-i}(M), i=1,2, \ldots, n-1,
$$

together with suitable multilinear maps denoted collectively by $\ell$. The suffix 'Ham' refers to the Hamiltonian $(n-1)$-forms, that is, those forms $H$ such that

$$
\iota_{X} \omega+d H=\alpha(X)+d H=0
$$

for a vector field $X$ preserving $\omega$ (that is, $\mathcal{L}_{X} \omega=0$ ), called, in turn, a Hamiltonian vector field pertaining to $H$.

A form $\beta$ is said to be strictly (respectively, globally; respectively, locally) conserved by an $\omega$-preserving vector field $X$ if $\mathcal{L}_{X} \beta=0$ (respectively, $\mathcal{L}_{X} \beta$ is exact; respectively, $\mathcal{L}_{X} \beta$ is closed). Cartan's formula immediately shows that closed forms are globally conserved; indeed, for such a form,

$$
\mathcal{L}_{X} \beta=d \iota_{X} \beta+\iota_{X} d \beta=d \iota_{X} \beta .
$$

Recall, from [46], that a homotopy co-momentum map is an $L_{\infty}$-algebra morphism stemming from what is called an infinitesimal action of $\mathfrak{g}$ on $M$ (with $\mathfrak{g}$ being the Lie algebra of a generic Lie group $G$, acting on $M$ by $\omega$-preserving vector fields),

$$
(f): \mathfrak{g} \rightarrow L_{\infty}(M, \omega),
$$

given explicitly by a sequence of linear maps

$$
(f)=\left\{f_{i}: \Lambda^{i} \mathfrak{g} \rightarrow \Lambda^{n-i}(M) \mid 0 \leq i \leq n+1\right\}
$$

fulfilling $f_{0}=f_{n+1}=0$ (we have tacitly set $\Lambda^{-1}(M)=0$ ) and

$$
\operatorname{Im} f_{1} \in \Lambda_{\text {Ham }}^{1}(M)
$$

together with (for $p \in \Lambda^{k}(\mathfrak{g})$ )

$$
-f_{k-1}(\partial p)=d f_{k}(p)+\varsigma(k) \iota\left(v_{p}\right) \omega
$$

$(k=1, \ldots, n+1)$. We explain the notation. If $p=\xi_{1} \wedge \xi_{2} \wedge \cdots \wedge \xi_{k}$, then $v_{p}=v_{1} \wedge$ $v_{2} \wedge \cdots \wedge v_{k}$ where $v_{i} \equiv v_{\xi_{i}}$ are the fundamental vector fields associated to the action of $G$ on $M$. One sets $\iota\left(v_{p}\right) \omega=\iota\left(v_{k}\right) \cdots \iota\left(v_{1}\right) \omega, \varsigma(k):=-(-1)^{k(k+1) / 2}$ and defines $\partial \equiv \partial_{k}$ : $\Lambda^{k} \mathfrak{g} \rightarrow \Lambda^{k-1} \mathfrak{g}$ via

$$
\partial\left(\xi_{1} \wedge \xi_{2} \wedge \cdots \wedge \xi_{k}\right):=\sum_{1 \leq i<j \leq k}(-1)^{i+j}\left[\xi_{i}, \xi_{j}\right] \wedge \xi_{1} \wedge \cdots \hat{\xi}_{i} \wedge \cdots \wedge \hat{\xi}_{j} \wedge \cdots \xi_{k}
$$

(with` denoting deletion as usual and with $\partial_{0}=0$; one has $\partial^{2}=0$ ). 
Formula (2-2) tells us that the closed forms

$$
\mu_{k}:=f_{k-1}(\partial p)+\varsigma(k) \iota\left(v_{p}\right) \omega
$$

must actually be exact, with potential $-f_{k}(p)$. Closure can be quickly ascertained as follows (in view of Lemma 2.16 in [46] and keeping in mind that $d \omega=0$ ):

$$
\begin{aligned}
d\left(f_{k-1}(\partial p)+\varsigma(k) \iota\left(v_{p}\right) \omega\right) & =\varsigma(k)(-1)^{k} \iota\left(v_{\partial p}\right) \omega-\varsigma(k-1) \iota\left(v_{\partial p}\right) \omega \\
& \equiv[-\varsigma(k+1)-\varsigma(k-1)] \iota\left(v_{\partial p}\right) \omega \\
& =0,
\end{aligned}
$$

since in general $\varsigma(k) \varsigma(k+2)=-1$. Notice that the special case $k=n+1$ asserts that the function $\mu_{n+1}(\cdot)$ is constant, and its value is fixed by the condition

$$
f_{n}(\partial p)+\varsigma(n+1) \iota\left(v_{p}\right) \omega=0 .
$$

This can be rephrased, upon resorting to [7, Section 9] (we use a different notation), by asserting that the $\mathrm{g}-(n+1)$-cocycle

$$
c_{x}\left(p=\xi_{1} \wedge \xi_{2} \wedge \cdots \wedge \xi_{n+1}\right)=\left.\iota_{v_{p}} \omega\right|_{x}
$$

in the Chevalley-Eilenberg cochain (CE) complex $C E(\mathfrak{g})$ ought to be a boundary,

$$
c_{x}=\delta_{\mathrm{CE}}(b) \text {, }
$$

for a fixed but generic point $x \in M$ (the class $\left[c_{x}\right]$ being in general independent of $x \in M$ [7, Cor. 9.3]); the operator $\delta_{\mathrm{CE}}$ is the CE-differential defined by duality, $\left(\delta_{\mathrm{CE}} \phi\right)(p):=$ $\phi(\partial p), \phi \in C E(\mathfrak{g})$, and extended by linearity. Independence of $x$ is expressed via the formula [7, Prop. 9.1]

$$
c_{x^{\prime}}-c_{x}=\delta_{\mathrm{CE}}(b)
$$

where

$$
b\left(v_{1} \wedge v_{2} \wedge \cdots \wedge v_{n}\right):=-\varsigma(n+1) \int_{\gamma} \iota\left(v_{1} \wedge v_{2} \wedge \cdots \wedge v_{n}\right) \omega
$$

and $\gamma$ is a path connecting $x$ to $x^{\prime}$ (recall that $M$ is assumed to be connected).

We resume the above discussion in Section 2.3.

2.2. The hydrodynamical Poisson bracket. In the present subsection we briefly review, for motivation and further applications, the symplectic geometrical portrait underlying the theory of perfect fluids, in its simplest instance. We denote by $\mathfrak{g}$ the (infinite-dimensional) Lie subalgebra of $\mathfrak{X}\left(\mathbb{R}^{3}\right)$ consisting of the divergence-free vector fields on $\mathbb{R}^{3}$ (the 'Lie algebra' of the 'Lie group' $G=\operatorname{sDiff}\left(\mathbb{R}^{3}\right)$ of volume-preserving diffeomorphisms of $\mathbb{R}^{3}$. As is often done, we gloss over analytic subtleties; see, for example, [2, 3, 13, 29] for more information). We just recall here that $G$ is a regular Lie group in the sense of Kriegl and Michor [29, 38.4] and that its associated exponential map is not even locally surjective (a quite general phenomenon). We also tacitly assume that our fields rapidly vanish at infinity, so that convergence problems 
are avoided and boundary terms are absent. The 'hydrodynamical' Lie bracket, given by $\left[\xi_{1}, \xi_{2}\right]=\operatorname{curl}\left(\xi_{1} \times \xi_{2}\right)$ and equalling minus the standard one, is employed throughout.

Also, following [3], for example, we consider the so-called regular dual $\mathfrak{g}^{*}$ of $\mathfrak{g}$ consisting of all 1-forms modulo exact 1 -forms,

$$
\mathfrak{g}^{*}:=\Lambda^{1}\left(\mathbb{R}^{3}\right) / d \Lambda^{0}\left(\mathbb{R}^{3}\right),
$$

together with the standard pairing $\left(\omega \in \mathfrak{g}^{*}, \xi \in \mathfrak{g}\right)$

$$
(\omega, \xi)=\int\langle\omega(x), \xi(x)\rangle d^{3} x .
$$

Nevertheless, we feel free to use suitable genuine distributional elements as well (i.e. currents in the sense of de Rham, [12]) from the full topological dual (without introducing new notation for the latter). Everything is clear from the context.

The (regular) dual $\mathrm{g}^{*}$ is naturally interpreted as a Poisson manifold with respect to the hydrodynamical Poisson bracket (Arnol'd-Marsden-Weinstein Lie-Poisson structure),

$$
\{F, G\}([\mathbf{v}])=\int_{\mathbb{R}^{3}}\left\langle\mathbf{v},\left[\frac{\delta F}{\delta \mathbf{v}}, \frac{\delta G}{\delta \mathbf{v}}\right]\right\rangle d^{3} x,
$$

with $\mathbf{v} \in \mathfrak{g}$ (velocity field), $\mathbf{w}:=$ curl $\mathbf{v}$ its vorticity, with $[\mathbf{v}]$ denoting the 'gauge' class of $\mathbf{v}:[\mathbf{v}]=\{\mathbf{v}+\nabla f\}$; see, for example, [3, 30, 32, 36-38, 49]. The Euler evolution, in its so-called vorticity form

$$
\frac{\partial \mathbf{w}}{\partial t}+[\mathbf{w}, \mathbf{v}]=0
$$

is volume-preserving and also preserves the symplectic leaves of $\mathfrak{g}^{*}$ given by the G-coadjoint orbits $O_{[\mathrm{v}]} \equiv O_{\mathrm{w}}$. The symplectic structure on $O_{\mathrm{w}}$ is the Kirillov-Kostant-Souriau (KKS) one [27, 28, 47]:

$$
\Omega_{\mathrm{KKS}}([\mathbf{v}])\left(\operatorname{ad}_{\mathbf{b}}^{*}([\mathbf{v}]), \operatorname{ad}_{\mathbf{c}}^{*}([\mathbf{v}])\right)=\int_{\mathbb{R}^{3}}\langle\mathbf{v},[\mathbf{b}, \mathbf{c}]\rangle d^{3} x=\int_{\mathbb{R}^{3}}\langle\mathbf{w}, \mathbf{b} \times \mathbf{c}\rangle d^{3} x
$$

with the coadjoint action reading, explicitly, up to a gradient (not influencing calculations),

$$
\operatorname{ad}_{\mathbf{b}}^{*}(\mathbf{v})=-\mathbf{w} \times \mathbf{b} \quad\left(\equiv \operatorname{ad}_{\mathbf{b}}^{*}([\mathbf{v}])\right) .
$$

The Hamiltonian algebra $\Lambda$ pertaining to $O_{w}$ consists of the so-called Rasetti-Regge currents originally introduced in [40] and further developed in [6, 36-38, 49]):

$$
\lambda_{\mathbf{b}}(\mathbf{v})=\int\langle\mathbf{b}, \mathbf{v}\rangle=\int\langle\mathbf{B}, \mathbf{w}\rangle
$$

(with curl $\mathbf{B}=\mathbf{b}$ ), fulfilling, for $\mathbf{b}, \mathbf{c} \in \mathfrak{g}$,

$$
\left\{\lambda_{\mathbf{b}}, \lambda_{\mathbf{c}}\right\}=\lambda_{[\mathbf{b}, \mathbf{c}]},
$$


that is, the map

$$
\mathfrak{g} \ni \mathbf{b} \mapsto \lambda_{\mathbf{b}} \in \Lambda
$$

is a G-equivariant co-momentum map (observe, in particular, that $\delta \lambda_{\mathbf{b}} / \delta \mathbf{v}=\mathbf{b}$ ).

The preceding portrait carries through to the singular vorticity case, in particular when the vorticity field is $\delta$-like and concentrated on a two-dimensional patch, filament or loop. Dealing with the latter case, we ultimately retrieve the Brylinski manifold $Y$ consisting of smooth oriented knots (smooth embedded loops modulo orientation-preserving reparametrizations) together with its symplectic structure $\Omega_{Y}$ and the original Rasetti-Regge currents (see [4, 6] for more details):

$$
\Omega_{Y}(\cdot, \cdot)(\gamma):=\int_{\gamma} v(\dot{\gamma}, \cdot, \cdot)=\int_{\gamma}\langle\dot{\gamma}, \cdot \times \cdot\rangle, \quad \lambda_{\mathbf{b}}(\gamma):=\int_{\gamma} \mathbf{B} .
$$

Indeed, recall that, given a volume form $v$ on a three-dimensional $M$, one gets, by transgression, a 2-form $\Omega$ on $L M$ via the formula

$$
\Omega=\iota_{V} \int_{S^{1}} \operatorname{ev}^{*}(v)
$$

where ev : $L M \times S^{1} \rightarrow M$ given by $\operatorname{ev}(\gamma, t):=\gamma(t)$ is the evaluation map of a loop $\gamma \in$ $L M$ at a point $t \in S^{1} \equiv[0,1] / \sim$ (endpoint identification), and $\iota_{V}$ denotes contraction with the vector field on $L M$ given by $\left.V\right|_{\gamma}=\dot{\gamma}$. More explicitly, given tangent vectors $u$ and $v$ at $\gamma$, the symplectic form reads (cf. [6], formula 6-8, p. 238):

$$
\Omega_{\gamma}(u, v)=\int_{0}^{1} v_{\gamma(t)}(\dot{\gamma}(t), u(t), v(t)) d t
$$

(where we set $\dot{\gamma}=d \gamma / d t$ ). The above construction carries through to $Y$. In this case, the coadjoint orbits are labelled by the equivalence types of knots (via ambient isotopies), by virtue of a result of Brylinski; see [6].

2.3. A hydrodynamical homotopy co-momentum map. In this subsection we elaborate on the previous discussion by introducing an explicit homotopy co-momentum map, departing from the standard setting since our group $G=\operatorname{sDiff}\left(\mathbb{R}^{3}\right)$ is infinite-dimensional. We start from the observation [8] that the volume form in $\mathbb{R}^{3}$, $v:=d x \wedge d y \wedge d z$, can be interpreted as a multisymplectic form: in this case the map $\alpha$ is bijective (in particular, injective). In coordinates, if $\xi=\left(\xi^{i}\right)$, then

$$
\alpha(\xi)=\iota_{\xi} v=\xi^{1} d y \wedge d z+\xi^{2} d z \wedge d x+\xi^{3} d x \wedge d y .
$$

Upon introducing the Hodge $*$ relative to the standard Euclidean metric and the associated 'musical isomorphisms', we have $\left(\xi \in \mathfrak{X}\left(\mathbb{R}^{3}\right), \beta \in \Lambda^{2}\left(\mathbb{R}^{3}\right)\right)$

$$
\alpha(\xi)=*\left(\xi^{b}\right), \quad \alpha^{-1}(\beta)=(* \beta)^{\sharp} .
$$

Then we have, for $\xi \in \mathfrak{g}$ (via Cartan's formula),

$$
0=\mathcal{L}_{\xi} v=d \iota_{\xi} v+\iota_{\xi} d v=d \iota_{\xi} v=\operatorname{div}(\xi) v
$$


and thus we have an isomorphism $g \cong Z^{2}\left(\mathbb{R}^{3}\right)$ (closed 2-forms on $\mathbb{R}^{3}$ ). This is important in the sequel. The above also expresses the fact that $v$ is a strictly conserved 3 -form.

We now give the promised example of homotopy co-momentum map emerging in fluid dynamics. Define, for $\mathbf{b} \in \mathfrak{g}$,

$$
f_{1}(\mathbf{b}):=-B,
$$

where $B=\mathbf{B}^{b}$ and $\mathbf{B}$ is again a vector potential for $\mathbf{b}$, that is, $\operatorname{curl} \mathbf{B}=\mathbf{b}$, chosen, for example, in such a way that $\operatorname{div} \mathbf{B}=0$ (Coulomb gauge).

It can immediately be checked that

$$
d f_{1}(\mathbf{b})+\iota_{\mathbf{b}} v=d f_{1}(\mathbf{b})+\alpha(\mathbf{b})=0 .
$$

The above formula tells us that $f_{1}(\mathbf{b})$ is a Hamiltonian 1-form for $\mathbf{b}$ (and, conversely, that the vector field $\mathbf{b}$ is a Hamiltonian vector field pertaining to $f_{1}(\mathbf{b})$, in accordance with (2-1) in Section 2.1). Any $f_{1}(\mathbf{b})$ above is also a Noether current in the sense of Gotay et al. [20]. In order to complete the definition of a homotopy co-momentum map, we just have to find $f_{2}$ satisfying formula (2-2) above. Indeed, for $k=1$ we retrieve (2-8). The case $k=2$ reads

$$
-f_{1}(\partial p)=d f_{2}(p)+\iota_{v_{p}} v
$$

For $\xi_{i} \in \mathfrak{g}(i=1,2)$, let $p=\xi_{1} \wedge \xi_{2}$, so $\partial p=-\left[\xi_{1}, \xi_{2}\right]$.

Then one checks (using, for example, [46, Lemma 2.18], or the preceding subsection) that

$$
d f_{1}\left(\left[\xi_{1}, \xi_{2}\right]\right)=d\left(\iota_{\xi_{1} \wedge \xi_{2}} v\right)=-\iota_{\left[\xi_{1}, \xi_{2}\right]} v
$$

(recall that $\left.\iota_{\xi_{1} \wedge \xi_{2}} v=v\left(\xi_{1}, \xi_{2}, \cdot\right)\right)$. Therefore, the 1-form $\mu_{2}\left(\xi_{1}, \xi_{2}\right):=f_{1}\left(\left[\xi_{1}, \xi_{2}\right]\right)-$ $\iota_{\xi_{1} \wedge \xi_{2}} v$ is closed, hence exact, and (2-9) tells us that $f_{2}(p)$ is a potential for it and, as such, is determined up to a constant $c\left(\xi_{1}, \xi_{2}\right)$. In order to prove that we have a bona fide co-momentum map, we must have, in particular, for $q=\xi_{1} \wedge \xi_{2} \wedge \xi_{3}$, the explicit formula

$$
f_{2}(\partial q)=v\left(\xi_{1}, \xi_{2}, \xi_{3}\right)
$$

which is a priori true up to a constant $c\left(\xi_{1}, \xi_{2}, \xi_{3}\right)$ by virtue of (2-9) and [7, Lemma 9.2]. However, the constant is in fact zero since $v\left(\xi_{1}, \xi_{2}, \xi_{3}\right)$ vanishes at infinity, and the same is true for $f_{2}(\partial q)$ upon solving the related Poisson equation

$$
\Delta f_{2}(\partial q)=\Delta v\left(\xi_{1}, \xi_{2}, \xi_{3}\right)
$$

(obtained via a straightforward computation; notice that we use the Riemannian Laplacian, which is minus the standard one).

An alternative derivation uses $x$-independence of the class $\left[c_{x}\right]$. Upon taking $S^{3}=$ $\mathbb{R}^{3} \cup\{\infty\}$, we have $c_{\infty}=0$, hence $c_{x}=\delta_{\mathrm{CE}}(b)$, with

$$
b=-\int_{\gamma_{\infty}} \iota\left(v_{1} \wedge v_{2}\right) v
$$


( $\gamma_{\infty}$ being a path connecting $x$ to $\infty$, cf. (2-3): the expression is meaningful in view of the assumed decay at infinity of our objects). This is equivalent to the previous equation (2-10). The function $f_{2}$ is the given by the solution of a Poisson equation,

$$
f_{2}=\Delta^{-1} \delta \mu_{2}
$$

(in the present case $\delta=-* d *$ ). This completes the construction of the required homotopy co-momentum map.

We define Poisson brackets via the expression

$$
\left\{f_{1}(\mathbf{b}), f_{1}(\mathbf{c})\right\}(\cdot):=\iota_{\mathbf{c}} \iota_{\mathbf{b}} v(\cdot)=v(\mathbf{b}, \mathbf{c}, \cdot)
$$

which is employed below and in Section 4.

We may also naturally ask the question whether the above map $(f)$ is (infinitesimally) G-equivariant, in the sense of [46]: in particular, one should check the validity of the formula

$$
\mathcal{L}_{\xi} f_{1}(\mathbf{b})=f_{1}([\xi, \mathbf{b}])
$$

for all $\xi, \mathbf{b} \in \mathfrak{g}$. However, working out the two sides of the above equation easily yields, in particular, for $\xi=\mathbf{b}$, the equality

$$
d B(\mathbf{b})=0,
$$

that is, in vector terms $\langle\mathbf{B}, \mathbf{b}\rangle=c=0$ since $\mathbf{b}$ is compactly supported. However, if one considers a flux tube with nonzero helicity $\int\langle\mathbf{B}, \mathbf{b}\rangle$ (see $[4,35,48]$ and below for further elucidation of this train of concepts), we get a contradiction. Notice that the argument does not depend on the choice of $B$. The lack of $G$-equivariance is not surprising, since our construction involves Riemannian geometric features. We may now recap the preceding discussion via the following theorem.

\section{THEOREM 2.1.}

(i) The map $(f)$ previously given through the above $f_{j}: \Lambda^{j} \mathfrak{g} \rightarrow \Lambda^{2-j}\left(\mathbb{R}^{3}\right)$, fulfilling (2-8), (2-5), and (2-6), yields a homotopy co-momentum map; explicitly,

$$
f_{1}=f_{1}(\mathbf{b}):=-B,\left(f_{1}=b \circ \operatorname{curl}^{-1}\right) ; \quad f_{2}=\Delta^{-1} \delta \mu_{2} .
$$

(ii) The above homotopy co-momentum map transgresses, via the evaluation map ev $: L \mathbb{R}^{3} \times \mathbb{R} \ni(\gamma, t) \mapsto \gamma(t) \in \mathbb{R}^{3}$, to the hydrodynamical co-momentum map of Arnol'd, Marsden and Weinstein, defined on the Brylinski manifold Y of oriented knots (cf. Section 2.2).

(iii) Moreover, we have the formula

$$
\left\{f_{1}(\mathbf{b}), f_{1}(\mathbf{c})\right\}-f_{1}([\mathbf{b}, \mathbf{c}])=-d f_{2}(\mathbf{b} \wedge \mathbf{c}) .
$$

(iv) The map $(f)$ is not G-equivariant in the sense of Ryvkin-Wurzbacher-Zambon [46]. 
PROOF. We need only address point (ii): observe that the relevant piece of the homotopy co-momentum map is $f_{1}$ which, under transgression, becomes

$$
\mu_{\mathbf{b}}=-\int_{\gamma} B=-\lambda_{\mathbf{b}}
$$

that is, up to sign, the Rasetti-Regge current $\lambda_{\mathbf{b}}$ pertaining to $\mathbf{b} \in \mathfrak{g}$ independent of the choice of $B$. This is in accordance with the general result in [46] asserting that, roughly speaking, homotopy co-momentum maps transgress to homotopy co-momentum maps on loop (and even mapping) spaces. Actually, the ansatz for the $f_{1}$ term was precisely motivated by this phenomenon.

Formula (2-12) in (iii) is just a rewriting of (2-9).

2.4. A generalization to Riemannian manifolds. We should notice that a hydrodynamically flavoured homotopy co-momentum map can be similarly construed also for an $(n+1)$-dimensional connected, compact, orientable Riemannian manifold $(M, g)$, upon taking its Riemannian volume form $v$ as a multisymplectic form and again the group $G$, consisting of all volume-preserving diffeomorphisms, as symmetry group. The divergence of a vector field $X$ is defined via $\operatorname{div} X:=* d * X^{\mathrm{b}}=-\delta X^{\mathrm{b}}$; the operator $*$ is the Riemannian Hodge star (e.g. [29, 51]). We can indeed prove the following result.

THEOREM 2.2. Let $(M, g)$ be a connected compact oriented Riemannian manifold of dimension $n+1, n \geq 1$, with multisymplectic form $v$ given by its Riemannian volume form, and such that the de Rham cohomology groups $H_{\mathrm{dR}}^{k}(M)$ vanish for $k=1,2, \ldots n-1$ (one necessarily has $H_{\mathrm{dR}}^{0}(M)=H_{\mathrm{dR}}^{n+1}(M)=\mathbb{R}$ ). Let $\mathrm{g}_{0}$ be the Lie subalgebra of $\mathrm{g}$ consisting of divergence-free vector fields vanishing at a point $x_{0} \in M$. Then there exists an associated family of $\mathrm{g}_{0}$-homotopy co-momentum maps.

PROOF. As we have already noticed in general, the defining formula triggers a recursive construction starting from $f_{1}$, up to topological obstructions (we have a sequence of closed forms, which must actually be exact, together with the constraint $f_{n}(\partial q)=(-1)^{(n+1)(n+2) / 2} v\left(\xi_{1}, \ldots \xi_{n+1}\right)$, with $q=\xi_{1} \wedge \cdots \xi_{n+1}$, for the constant function $\left.\mu_{n+1}(\cdot)\right)$. In the present case, a natural candidate for the $(n-1)$-form $f_{1}$ can be readily manufactured via Hodge theory (see, for example, [51]):

$$
f_{1}(\xi):=-\Delta^{-1} \delta\left(\iota_{\xi} v\right)
$$

(the direct generalization of the preceding case) after imposing $\delta f_{1}(\xi)=0$ (the analogue of the Coulomb gauge condition), provided one can safely invert the Hodge Laplacian $\Delta=d \delta+\delta d$, this being the case if $H_{\mathrm{dR}}^{n-1}(M)=0$. One can of course alter the above definition by addition of an exact form. The topological assumptions made ensure that the entire procedure goes through unimpeded due to the formula

$$
d f_{k}\left(\xi_{1} \wedge \cdots \wedge \xi_{k}\right)=\mu_{k}\left(\xi_{1} \wedge \cdots \wedge \xi_{k}\right), \quad k=2,3, \ldots n
$$


(see Equation (2-3)). Finally, one needs to check that

$$
f_{n}\left(\partial\left(\xi_{1} \wedge \cdots \wedge \xi_{n+1}\right)\right)=-\varsigma(n+1) \iota\left(\xi_{1} \wedge \cdots \wedge \xi_{n+1}\right) \nu,
$$

but this is true once we observe that, since $c_{x_{0}}=0$, the class $\left[c_{x}\right]=0$ (cf. Section 2.1). Therefore, we eventually have the compact formulae

$$
f_{1}(\xi):=-\Delta^{-1} \delta\left(\iota_{\xi} v\right) ; \quad f_{k}=\Delta^{-1} \delta \mu_{k}, \quad k=2, \ldots, n .
$$

REMARK 2.3. We notice that the above result holds, in particular, for homology spheres such as the celebrated Poincaré dodecahedral space. We point out that the case in which the intermediate homology groups are at most torsion (hence not detectable by de Rham techniques) is also encompassed: this is, for example, the case of lens spaces. Notice that $G$-equivariance cannot be expected a priori. Also notice that one could restrict to the natural symmetry group provided by the isometries of $(M, g)$. See, for example, [44] for a general discussion of topological constraints to existence and uniqueness of homotopy co-momentum maps.

2.5. On weak homotopy moment maps. We should observe that any homotopy co-momentum map induces a weak homotopy moment map (see, for example, [24, 31]): if in (2-2) we set $\partial p=0$, we get

$$
d f_{k}(p)+\varsigma(k) \iota\left(v_{p}\right) \omega=0
$$

for $k=1, \ldots, n$, which is the very property defining a weak homotopy moment map. The paper [24] (Theorems 1.2-6) shows that such maps allow for direct generalizations of the classical momentum map obstruction results. It would be interesting to adapt this setting to our infinite-dimensional group situation. For the time being we just observe that, in Theorem 2.2 above, upon relaxing the condition that our fields vanish at a point, we get a weak homotopy moment map which, in general, is not a homotopy co-momentum map since we drop condition (2-14).

2.6. Covariant phase space aspects. We now propose a multisymplectic interpretation of the hydrodynamical bracket which ties in neatly with the topics discussed in previous sections, via covariant phase space ideas [11, 15, 20, 26, 52], but without literally following the standard recipe, as we see shortly.

Starting with a four-dimensional space-time $M=\mathbb{R}^{3} \times \mathbb{R} \leadsto(x, y, z, t)$, define the obvious trivial bundle

$$
E=M \times \mathbb{R}^{3} \rightarrow M
$$

and interpret $\Sigma:=\mathbb{R}^{3} \rightsquigarrow(x, y, z)$ as a Cauchy 'submanifold' of $M$.

Any divergence-free vector field can be viewed as an initial condition $\mathbf{v}(x, 0)$ for the (volume-preserving) Euler evolution (at least for small times, but as we previously said, we do not insist on refined analytical nuances) $\mathbf{v}(x, t)$, yielding a section of $E$. Using the 3-volume form $v$, orienting fibres (notice that, when viewed on $E$, it is only pre-2-plectic, which is to say closed but degenerate), and observing that we can set

$$
\mathcal{J}^{1} \mathbf{v}:=\mathbf{w} \quad(:=\operatorname{curl} \mathbf{v})
$$


(the natural "covariant; jetification of the section $\mathbf{v}$-to be distinguished from the standard jetification $j^{1}$ - if we wish to look at $\mathbf{v}$ as the vector space counterpart of a connection 1-form, yielding a section of the jet bundle $J^{1} E \rightarrow E$ ), we can rewrite the hydrodynamical bracket, mimicking [15], as

$$
\{F, G\}([\mathbf{v}])=\int_{\Sigma=\mathbb{R}^{3}}\left\langle\mathbf{w}, \frac{\delta F}{\delta \mathbf{v}} \times \frac{\delta G}{\delta \mathbf{v}}\right\rangle d^{3} x=\int_{\Sigma=\mathbb{R}^{3}} v\left(\mathcal{J}^{1} \mathbf{v}, \frac{\delta F}{\delta \mathbf{v}}, \frac{\delta G}{\delta \mathbf{v}}\right) d^{3} x=:(\star)
$$

since the variations $\delta F / \delta \mathbf{v}$ and $\delta G / \delta \mathbf{v}$ are vertical and divergence-free: $\delta F / \delta \mathbf{v}=$ $\operatorname{curl}(\delta F / \delta \mathbf{w})$. Taking again $\mathbf{b}=\operatorname{curl} \mathbf{B}$ etc. and finally setting $F=\lambda_{\bullet}$ (see, for example, $[37,49])$, we see that the expression $(\star)$ can be manipulated to yield the expressive layout (with slight abuse of language)

$$
(\star)=\int_{\Sigma}\left(\mathcal{J}^{1^{*}} v\right)(\mathbf{v}, \mathbf{B}, \mathbf{C}) d^{3} x=\int_{\Sigma} v\left(\mathcal{J}^{1} \mathbf{v}, \mathcal{J}^{1} \mathbf{B}, \mathcal{J}^{1} \mathbf{C}\right) d^{3} x=\int_{\Sigma} v(\mathbf{w}, \mathbf{b}, \mathbf{c}) d^{3} x
$$

(in full adherence with the discussion in Section 2.2). The same portrait can be painted, mutatis mutandis, for the singular case. Ultimately, we reach the following conclusion.

THEOREM 2.4. (i) The Poisson manifold $\mathrm{g}^{*}$ can be naturally be interpreted as a (generalized) covariant phase space pertaining to the volume-preserving Euler evolution: the latter indeed preserves the symplectic leaves of $\mathrm{g}^{*}$ given by the G-coadjoint orbits $\mathcal{O}_{[\mathrm{v}]}$.

(ii) The above construction reproduces the symplectic structure of $Y$ upon taking singular vorticities, concentrated on a smooth oriented knot: the covariant phase space picture is fully retrieved upon passing to a two-dimensional space-time $S^{1} \times \mathbb{R} \rightsquigarrow$ $(\lambda, t)$, with $\lambda \in S^{1} \equiv \Sigma$ being a knot parameter (and staying of course with the same $v$ ).

REMARK 2.5. We stress the fact that we did not literally follow the standard 'multisymplectic to covariant' recipe developed in [15]. In fact the multisymplectic manifold we consider is not the one prescribed by [20] since we directly took the standard volume form $v$ on $\mathbb{R}^{3}$ as a 2-plectic structure (or pre-2-plectic when pulled back to $E$ ); cf. [8]. This neatly matches Brylinski's theory and fits with the stance long advocated, among others, by Rasetti and Regge and by Goldin (see also, for example, [17-19, 40,49]), pinpointing the special and ubiquitous role played by the group $G$. Another motivation for considering $v$ is its pivotal role in the formulation of conservation theorems (see [46]). We pursue this aspect in what follows.

REMARK 2.6. In line with Remark 2.5, notice that the above description can, in principle, be generalized to any volume form (on an orientable manifold), with its attached group $G$. The covariant phase space picture should basically persist in the sense that one might construct, in greater generality, an $n$-plectic structure out of an $(n+1)$-plectic one via an expression akin to $(\star)$. The (non-) $G$-equivariance issue should be relevant in this context. 


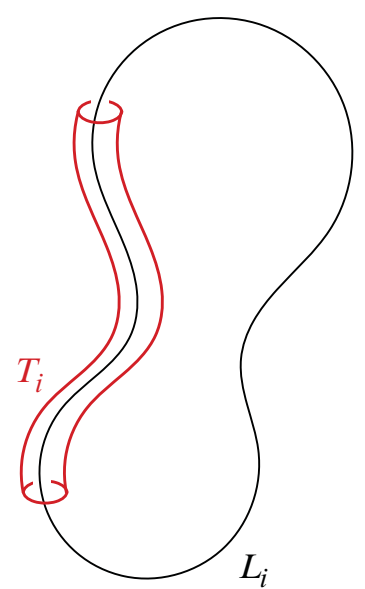

FIGURE 1. Tubular neighbourhoods.

\section{A Hamiltonian 1-form for links}

We may specialize the considerations in Section 2.3 to the case of links. The basic and quite natural idea is to associate to a knot (or link) a perfect fluid whose vorticity is concentrated thereon (cf. the preceding discussion on the Brylinski manifold). As general references for knot theory we quote, among others, [43], together with [5] for the algebraic-topological tools employed here. Recall that in general the Poincaré dual $\left[\eta_{S}\right] \in H^{n-k}(M)$ of a $k$-dimensional closed oriented submanifold $S$ of an $n$-dimensional manifold $M$ is characterized by the property

$$
\int_{M} \omega \wedge \eta_{S}=\int_{S} i^{*} \omega
$$

for any closed, compactly supported $k$-form $\omega$ on $M(i: S \hookrightarrow M$ being the inclusion map). We shall view Poincaré duals as either genuine forms or currents in the sense of de Rham [12].

Building on $[4,39,48]$, let $L=\bigcup_{i=1}^{n} L_{i}$ be an oriented link in $\mathbb{R}^{3}$ with components $L_{i}, i=1, \ldots, n$ (required to be trivial knots) and let $\omega_{L_{i}}$ denote the Poincaré (or Thom) dual (class) associated to $L_{i}$ : they are 2-forms localized in a cross-section of a suitable tubular neighbourhood $T_{i}$ around $L_{i}$, with total fibre integral equal to one (see [5]), or, as currents, 2-forms which are $\delta$-like on $L_{i}$ (see Figure 1).

Then take, for each $i=1,2, \ldots, n$, a 1 -form $v_{L_{i}}$ such that $d v_{L_{i}}=\omega_{L_{i}}$, that is, $v_{L_{i}}:=$ $\omega_{\mathfrak{a}_{i}}$ is the Poincaré dual (class) of a disc $\mathfrak{a}_{i}$ bounding $L_{i}$ (a Seifert surface for the trivial knot $\left.L_{i}\right)$. Specifically,

$$
\partial \mathfrak{a}_{i}=L_{i}, \quad d v_{L_{i}}=d \omega_{\mathfrak{a}_{i}}=\omega_{L_{i}}=\omega_{\partial \mathfrak{a}_{i}} ;
$$



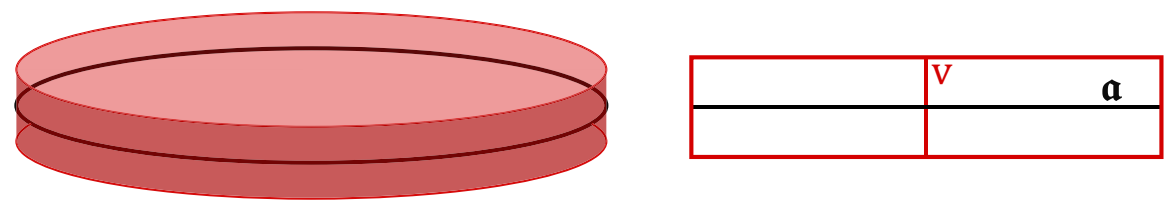

FIGURE 2. Poincaré duals.

see Figure 2. We list, for the sake of clarity, (de Rham) cohomology and relative homology groups of $S^{3} \backslash L$ with real coefficients, respectively:

$$
\begin{aligned}
& H^{0}\left(S^{3} \backslash L\right) \cong H_{3}\left(S^{3}, L\right) \cong \mathbb{R}, \\
& H^{1}\left(S^{3} \backslash L\right) \cong H_{2}\left(S^{3}, L\right) \cong \mathbb{R}^{n}, \\
& H^{2}\left(S^{3} \backslash L\right) \cong H_{1}\left(S^{3}, L\right) \cong \mathbb{R}^{n-1}, \\
& H^{3}\left(S^{3} \backslash L\right) \cong H_{0}\left(S^{3}, L\right) \cong 0 .
\end{aligned}
$$

The (de Rham classes of) the forms (or currents) $v_{L_{i}}$ in fact generate the cohomology group $H^{1}\left(S^{3} \backslash L, \mathbb{R}\right)$ (or, better, that of $S^{3} \backslash T$, with $T=\bigcup_{i=1}^{n} T_{i}$ ). Their homological counterparts are given by the (classes of) the discs $\mathfrak{a}_{i}$. One can also interpret the other groups: in particular, elements in $H_{1}\left(S^{3}, L\right)$ can be represented by classes $\left[\gamma_{i j}\right]$ of (smooth) paths $\gamma_{i j}$ connecting two components $L_{i}$ and $L_{j}$, subject to the relation $\left[\gamma_{i j}\right]+\left[\gamma_{j k}\right]=\left[\gamma_{i k}\right]$.

Now set

$$
\omega_{L}:=\sum_{i=1}^{n} \omega_{L_{i}}
$$

(the vorticity 2 -form for the link $L$ ) together with its velocity 1 -form

$$
v_{L}=\sum_{i=1}^{n} v_{L_{i}}, \quad d v_{L}=\omega_{L} .
$$

PROPOSITION 3.1. The position

$$
H=v_{L}
$$

produces a Hamiltonian 1-form for links.

PROOF. The proof is straightforward. Indeed, for each component $L_{i}$, the Hamiltonian vector field $\xi_{L_{i}}$ for $v_{L_{i}} \equiv v_{i}$ is minus the vector field associated to the closed 2-form $\omega_{L_{i}}$ (via the map $\alpha$ of Section 2). Explicitly, one has (setting $\xi_{L}=\sum_{i=1}^{n} \xi_{L_{i}}$ )

$$
d v_{L}+\iota_{\xi_{L}} v=0
$$


REMARK 3.2. Inspection of the very geometry of Poincare duality shows that the velocity 1 -forms $v_{i}$ correspond (upon approximation of the associated Euler equation) to the so-called linear induction approximation or binormal evolution of the "vortex ring' $L_{i}$ ('orthogonal' to the discs $\mathfrak{a}_{i}$-an easy depiction; cf. Figure 2); see [25] for more information. Formula (3-1) is the prototype for the calculations in Section 4.

Let us define the Chern-Simons (helicity) 3-form:

$$
C S(L):=v_{L} \wedge \omega_{L} .
$$

Integration of $C S(L)$ over $\mathbb{R}^{3}$ or $S^{3}$ yields an integer $\mathcal{H}(L)$, the helicity of $L$ :

$$
\int_{S^{3}} C S(L)=: \mathcal{H}(L)=\sum_{i, j=1}^{n} \ell(i, j),
$$

with $\ell(i, j)=\ell(j, i)$ being the Gauss linking number of components $L_{i}$ and $L_{j}$ if $i \neq j$ and $\ell(j, j)$ the framing of $L_{j}$, equal to $\ell\left(L_{j}, L_{j}^{\prime}\right)$ with $L_{j}^{\prime}$ being a section of the normal bundle of $L_{j}$; see, for example, [35, 41, 43, 48] and below. A regular projection of a link onto a plane produces a natural framing called the blackboard framing.

On a Riemannian 3-manifold $M$, the helicity $\mathcal{H}$ pertaining to a perfect fluid with velocity $\mathbf{v}$ and vorticity $\mathbf{w}=\operatorname{curl} \mathbf{v}$ is given (in notation as in the previous sections) by

$$
\mathcal{H}=\int_{M}\langle\mathbf{v}, \mathbf{w}\rangle=\int_{M} v \wedge d v
$$

(the last expression being the differential form counterpart). Concretely, the helicity can be viewed as a measure of the mutual knotting of two generic flow lines; see [3, 35-37, 48] for a more extensive discussion. We used this concept in Section 2.3 to prove the nonequivariance of the hydrodynamical homotopy co-momentum map.

\section{A multisymplectic interpretation of Massey products}

In this section we resort to the techniques developed in Sections 2 and 3 above and propose a reformulation of the so-called higher-order linking numbers in multisymplectic terms. Ordinary and higher-order linking numbers provide a quite useful tool for the investigation of Brunnian phenomena in knot theory: recall that a link is almost trivial or Brunnian if upon removing any component therefrom one gets a trivial link. They can be defined recursively in terms of Massey products, or equivalently, Milnor invariants, by the celebrated Turaev-Porter theorem (see [14, 23, 39, 48]). We review, briefly and quite concretely, the basic steps of the Massey procedure, read differential-geometrically as in $[23,39,48]$, presenting at the same time our novel multisymplectic interpretation thereof.

Let $L$ be an oriented link with three or more components $L_{j}$. The cohomological reinterpretation of the ordinary linking number $\ell(1,2)$ of two components $L_{1}$ and $L_{2}$, say, starts from consideration of the closed 2-form

$$
\Omega_{12}:=v_{1} \wedge v_{2}
$$


yielding the (integral) de Rham class

$$
\left\langle L_{1}, L_{2}\right\rangle:=\left[\Omega_{12}\right] \in H^{2}\left(S^{3} \backslash L\right) .
$$

The linking number $\ell(1,2)$ is nonzero precisely when $\left\langle L_{1}, L_{2}\right\rangle$, which in $H_{1}\left(S^{3}, L\right)$ equals $\ell(1,2)\left[\gamma_{12}\right]$, is nontrivial. If the latter class vanishes (that is, $\Omega_{12}$ is exact), we have

$$
d v_{12}+v_{1} \wedge v_{2}=d v_{12}+\Omega_{12}=0
$$

for some 1-form $v_{12}$. Now, assuming that all the ordinary mutual linking numbers of the components under consideration vanish, one can manufacture the closed 2-form (Massey product)

$$
\Omega_{123}=v_{1} \wedge v_{23}+v_{12} \wedge v_{3}
$$

yielding a third-order linking number (as a class):

$$
\left\langle L_{1}, L_{2}, L_{3}\right\rangle:=\left[\Omega_{123}\right] \in H^{2}\left(S^{3} \backslash L\right) .
$$

If the latter class vanishes, we find a 1 -form $v_{123}$ such that

$$
d v_{123}+v_{1} \wedge v_{23}+v_{12} \wedge v_{3}=d v_{123}+\Omega_{123}=0
$$

It is then easy to devise a general pattern, giving rise to forms $v_{I}, \Omega_{I}$ ( $I$ being a general multi-index). Actually, everything can be organized-via Chen's calculus of iterated path integrals $[9,10]$-in terms of sequences of nilpotent connections $\mathbf{v}^{(k)}$, $k=1,2 \ldots$, on a trivial vector bundle over $S^{3} \backslash L$ and their attached curvature forms $\mathbf{w}^{(k)}$ (ultimately, the $\Omega_{I} ;[22,39,48,50]$ ), everything stemming from the Cartan structure equation

$$
d \mathbf{v}^{(k)}+\mathbf{v}^{(k)} \wedge \mathbf{v}^{(k)}=\mathbf{w}^{(k)}
$$

together with the ensuing Bianchi identity

$$
d \mathbf{w}^{(k)}+\mathbf{v}^{(k)} \wedge \mathbf{w}^{(k)}-\mathbf{w}^{(k)} \wedge \mathbf{v}^{(k)}=0
$$

(the latter implying closure of the forms $\Omega_{I}$ ). In order to give a flavour of the general argument, start from the nilpotent connection $\mathbf{v}^{(1)}$ with its corresponding curvature $\mathbf{w}^{(1)}$ :

$$
\mathbf{v}^{(1)}=\left(\begin{array}{cccc}
0 & v_{1} & 0 & 0 \\
0 & 0 & v_{2} & 0 \\
0 & 0 & 0 & v_{3} \\
0 & 0 & 0 & 0
\end{array}\right), \quad \mathbf{w}^{(1)}=\left(\begin{array}{cccc}
0 & 0 & \Omega_{12}=v_{1} \wedge v_{2} & 0 \\
0 & 0 & 0 & \Omega_{23}=v_{2} \wedge v_{3} \\
0 & 0 & 0 & 0 \\
0 & 0 & 0 & 0
\end{array}\right) .
$$

Then proceed similarly with

$$
\mathbf{v}^{(2)}=\left(\begin{array}{cccc}
0 & v_{1} & v_{12} & 0 \\
0 & 0 & v_{2} & v_{23} \\
0 & 0 & 0 & v_{3} \\
0 & 0 & 0 & 0
\end{array}\right), \quad \mathbf{w}^{(2)}=\left(\begin{array}{cccc}
0 & 0 & 0 & \Omega_{123}=v_{1} \wedge v_{23}+v_{12} \wedge v_{3} \\
0 & 0 & 0 & 0 \\
0 & 0 & 0 & 0 \\
0 & 0 & 0 & 0
\end{array}\right)
$$

(we made use of $d v_{12}+\Omega_{12}=d v_{23}+\Omega_{23}=0$ ), and so on. 

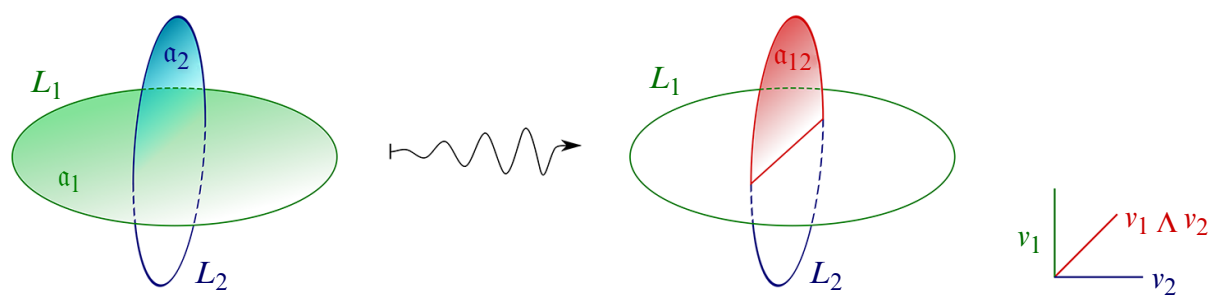

FIgURE 3. Starting the Chen procedure.

Also recall that all forms $\Omega_{I}$ can be neatly interpreted, via Poincaré duality, as auxiliary (trivial) knots $L_{I}$, and $v_{I}$ as discs bounded by $L_{I}$, in adherence to the considerations in Section 3; see [39, 48] for more details and worked-out examples, including the Whitehead link (involving fourth-order linking numbers with repeated indices) and the Borromean rings (exhibiting a third-order linking number). Just notice here that, for instance, formula (4-1) becomes, intersection-theoretically,

$$
\partial \mathfrak{a}_{12}+\mathfrak{a}_{1} \cap \mathfrak{a}_{2}=0 ;
$$

see Figure 3. Formula (4-2) can be rewritten as

$$
d v_{123}+\iota_{\xi_{123}} v=0
$$

where $\xi \equiv \xi_{123}=\alpha^{-1}\left(\Omega_{123}\right)$. The above ('vorticity') vector field $\xi_{123}$ can be thought of as being concentrated on the knot corresponding to $\xi_{123}$, or, alternatively, in a thin tube around it, when considering a bona fide Poincaré dual; cf. (3-1).

This tells us that $v_{123}$ is a Hamiltonian 1-form in the sense of [46], and the formula

$$
\mathcal{L}_{\xi} \Omega_{123}=d \iota_{\xi} \Omega_{123}+\iota_{\xi} d \Omega_{123}=d \iota_{\xi} \Omega_{123}
$$

expresses the fact that $\Omega_{123}$ is a globally conserved 2 -form, and the same holds for $\Omega_{12}$ and, in general, for $\Omega_{I}$, with their corresponding vector fields $\xi_{I}$. Specifically, we have the following proposition.

\section{PROPOSITION 4.1.}

(i) The volume form $v$ and all Massey 2-forms are globally conserved.

(ii) The 1-forms $v_{I}=f_{1}\left(\xi_{I}\right)$ are Hamiltonian with respect to the volume form.

\section{PROOF.}

(i) This is clear since the mentioned forms are closed.

(ii) The previous discussion can be rehearsed verbatim for a general multi-index $I$ :

$$
d v_{I}+\iota_{\xi_{I}} v=0
$$

(an extension of (3-1)), thus yielding the result.

The following is the main result of this section. 
THEOREM 4.2. In the above notation, the 1-forms $v_{I}$ are first integrals in involution with respect to the flow generated by the Hamiltonian vector field $\xi_{L}$, namely,

$$
\mathcal{L}_{\xi_{L}} v_{I}=0
$$

(that is, the $v_{I}$ are strictly conserved) and

$$
\left\{v_{I}, v_{J}\right\}=0
$$

(for multi-indices $I$ and $J$ ).

PROOF. Using Cartan's formula, we get

$$
\mathcal{L}_{\xi_{L}} v_{I}=d \iota_{\xi_{L}} v_{I}+\iota_{\xi_{L}} d v_{I}=d \iota_{\xi_{L}} v_{I}-\iota_{\xi_{L}} \iota_{\xi_{I}} v,
$$

but the second summand vanishes in view of the general expression

$$
\left\{v_{\xi}, v_{\eta}\right\}(\cdot)=v(\xi, \eta, \cdot)
$$

and of the peculiar structure of the vector fields involved (they either partially coincide or have disjoint supports). By the same argument, one gets $\iota_{\xi_{L}} v_{I}=0$, in view of the Poincare dual interpretation of $v_{I}$ (cf. Section 3), together with the second assertion; a crucial point to notice is that the auxiliary links obtained via Chen's procedure may be suitably split from their ascendants, this leading to

$$
\iota_{\xi_{L}} v_{I}=0,
$$

the consequent strict conservation of the $v_{I}$ being then immediate.

Notice that, in particular, from

$$
\iota_{\xi_{L}} v_{L}=0
$$

(Poincaré dual interpretation again) we also get

$$
\mathcal{L}_{\xi_{L}} v_{L}=0
$$

(this is not to be expected a priori in multisymplectic geometry; cf. [46]).

We remark that, upon altering the $v_{I}$ by an exact form, we may lose strict conservation, but in any case global conservation is assured (the Poisson bracket is an exact form, by (2-12) in Section 2 and in view of commutativity of the vector fields $\xi_{I}$ and $\left.\xi_{J}\right)$.

Ultimately, we can draw the conclusion that the Massey invariant route to ascertain the Brunnian character of a link can be mechanically understood as a recursive test of a kind of knot-theoretic integrability: the Massey linking numbers provide obstructions to the latter.

Thus, somewhat curiously, higher-order linking phenomena are interpreted in terms of multisymplectic geometry, which is a sort of higher-order symplectic geometry. Also, integrability comes in with a twofold meaning: first, higher-order linking numbers emerge from the construction of a sequence of flat, that is, integrable nilpotent connections; second, this very process yields first integrals in involution in a mechanical sense. 


\section{Conclusions and outlook}

In this note we have constructed a homotopy co-momentum map in a hydrodynamical context, whereby we gave, as an application, a multisymplectic reinterpretation of the Massey higher-order linking numbers, together with an extension thereof in a Riemannian geometric framework. We have also exhibited a covariant phase space interpretation of the geometrical framework involved.

The multisymplectic approach appears to be very promising for further advancement in this area. Also, the notion of integrability cropping up in our analysis of Massey products may deserve further scrutiny in a general multisymplectic context. We hope to tackle (at least some of) the open questions raised in this paper elsewhere.

\section{Acknowledgements}

The authors, both members of the GNSAGA group of INDAM, acknowledge support from Unicatt local D1-funds (ex MIUR 60\% funds). They are indebted to Tilmann Wurzbacher and Marco Zambon for enlightening discussions. They thank the referee for his/her careful reading and insightful comments. They are also grateful to Marcello Spera for help with graphics.

\section{References}

[1] R. Abraham and J. Marsden, Foundations of Mechanics, Benjamin/Cummings, Reading, MA, 1978.

[2] V. I. Arnol'd, 'Sur la géométrie différentielle des groupes de Lie de dimension infinie et ses applications à l'hydrodynamique des fluides parfaits', Ann. Inst. Fourier (Grenoble) 16 (1966) fasc. 1, 319-361.

[3] V. I. Arnol'd and B. Khesin, Topological Methods in Hydrodynamics (Springer, Berlin, 1998).

[4] A. Besana and M. Spera, 'On some symplectic aspects of knots framings', J. Knot Theory Ramifications 15 (2006), 883-912.

[5] R. Bott and L. Tu, Differential Forms in Algebraic Topology (Springer, Berlin, 1982).

[6] J.-L. Brylinski, Loop Spaces, Characteristic Classes and Geometric Quantization (Birkhäuser, Basel, 1993).

[7] M. Callies, Y. Frégier, C. L. Rogers and M. Zambon, 'Homotopy moment maps', Adv. Math. 303 (2016), 954-1043.

[8] F. Cantrijn, L. A. Ibort and M. De León, 'On the geometry of multisymplectic manifolds', J. Aust. Math. Soc. A 66(3) (1999), 303-330.

[9] K.-T. Chen, 'Iterated path integrals', Bull. Amer. Math. Soc. 83 (1977), 831-879.

[10] K.-T. Chen, Collected Papers of K.-T. Chen (eds. P. Tondeur and R. Hain) (Birkäuser, Boston, MA, 2001).

[11] Č. Crnković, 'Symplectic geometry of the covariant phase space', Classical and Quantum Gravity 5 (1988), 1557-1575.

[12] G. de Rham, Variétés Différentiables (Hermann, Paris, 1954).

[13] D. Ebin and J. Marsden, 'Groups of diffeomorphisms and the motion of incompressible fluids', Ann. of Math. 92 (1970), 102-163.

[14] R. A. Fenn, Techniques of Geometric Topology, London Mathematical Society Lecture Notes Series, 57 (Cambridge University Press, Cambridge, 1983).

[15] M. Forger and S. V. Romero, 'Covariant Poisson brackets in geometric field theory', Comm. Math. Phys. 256 (2005), 375-410. 
[16] Y. Frégier, C. Laurent-Gengoux and M. Zambon, 'A cohomological framework for homotopy moment maps', J. Geom. Phys. 97 (2015), 119-132.

[17] G. Goldin, 'Non-relativistic current algebras as unitary representations of groups', J. Math. Phys. 12 (1971), 462-488.

[18] G. Goldin, 'Parastatistics, $\theta$-statistics, and topological quantum mechanics from unitary representations of diffeomorphism groups', in: Proceedings of the XV International Conference on Differential Geometric Methods in Physics (eds. H. D. Doebner and J. D. Henning) (World Scientific, Singapore, 1987), 197-207.

[19] G. Goldin, 'Diffeomorphism groups and nonlinear quantum mechanics', J. Phys. Conf. Ser. 343 (2012), 012006.

[20] M. J. Gotay, J. Isenberg, J. E. Marsden and R. Montgomery, 'Momentum maps and classical fields. Part I: Covariant field theory', Preprint, 1998, arXiv:physics/9801019v2 [math-ph], 'Momentum maps and classical fields. Part II: Canonical analysis of field theories', Preprint, 2004, arXiv:math-phys/0411036 [math-ph].

[21] V. Guillemin and S. Sternberg, Symplectic Techniques in Physics (Cambridge University Press, Cambridge, 1984).

[22] R. Hain, 'The geometry of the mixed Hodge structure on the fundamental group', Proc. Sympos. Pure Math. 46 (1987), 247-282.

[23] J. J. Hebda and C. M. Tsau, 'An approach to higher order linking invariants through holonomy and curvature', Trans. Amer. Math. Soc. 364 (2012), 4283-4301.

[24] J. Herman, 'Existence and uniqueness of weak homotopy moment maps', J. Geom. Phys. 131 (2018), 52-65.

[25] B. Khesin, 'Topological fluid dynamics: theory and applications. The vortex filament equation in any dimension', Procedia IUTAM 7 (2013), 135-140.

[26] J. Kijowski and V. Szczyrba, 'A canonical structure for classical field theories', Comm. Math. Phys. 46 (1976), 183-206.

[27] A. Kirillov, 'Geometric quantization', in: Dynamical Systems IV, Encyclopaedia of Mathematical Sciences, 4 (Springer, Berlin, 2001), 139-176.

[28] B. Kostant, 'Quantization and unitary representations', in: Lectures in Modern Analysis and Applications, Lecture Notes in Mathematics, 170 (Springer, Berlin, 1970), pp. 87-208.

[29] A. Kriegl and P. W. Michor, The Convenient Setting of Global Analysis, Mathematical Surveys and Monographs, 53 (American Mathematical Society, Providence, RI, 1997).

[30] E. A. Kuznetsov and A. V. Mikhailov, 'On the topological meaning of canonical Clebsch variables', Phys. Lett. A 77 (1980), 37-38.

[31] L. Mammadova and L. Ryvkin, 'On the extension problem for weak moment maps', Preprint, 2020, arXiv:2001.00264.

[32] J. Marsden and A. Weinstein, 'Coadjoint orbits, vortices, and Clebsch variables for incompressible fluids', Physica 7 D (1983), 305-323.

[33] J. E. Marsden, S. Pekarsky, S. Shkoller and M. West, 'Variational methods, multisymplectic geometry and continuum mechanics', J. Geom. Phys. 38 (2001), 253-284.

[34] A. M. Miti and M. Spera, On some (multi)symplectic aspects of link invariants, Preprint, arXiv: 1805.01696 [math.DG] v2.

[35] H. K. Moffatt and R. L. Ricca, 'Helicity and the Călugăreanu invariant', Proc. R. Soc. Lond. A 439 (1992), 411-429.

[36] V. Penna and M. Spera, 'A geometric approach to quantum vortices', J. Math. Phys. 30 (1989), $2778-2784$.

[37] V. Penna and M. Spera, 'On coadjoint orbits of rotational perfect fluids', J. Math. Phys. 33 (1992), 901-909.

[38] V. Penna and M. Spera, 'String limit of vortex current algebra', Phys. Rev. B 62 (2000), 14547-14553.

[39] V. Penna and M. Spera, 'Higher order linking numbers, curvature and holonomy', J. Knot Theory Ramifications 11 (2002), 701-723. 
[40] M. Rasetti and T. Regge, 'Vortices in He-II, current algebras and quantum knots', Physica A 80 (1975), 217-233.

[41] R. L. Ricca and B. Nipoti, “"Gauss” linking number revisited', J. Knot Theory Ramifications 20 (2011), 1325-1343.

[42] C. L. Rogers, ' $L_{\infty}$-algebras from multisymplectic geometry', Lett. Math. Phys. 100 (2012), 29-50.

[43] D. Rolfsen, Knots and Links (Publish or Perish, Berkeley, CA, 1976).

[44] L. Ryvkin and T. Wurzbacher, 'Existence and unicity of co-moments in multisymplectic geometry', Differential Geom. Appl. 41 (2015), 1-11.

[45] L. Ryvkin and T. Wurzbacher, 'An invitation to multisymplectic geometry', J. Geom. Phys. 142 (2019), 9-36.

[46] L. Ryvkin, T. Wurzbacher and M. Zambon, 'Conserved quantities on multisymplectic manifolds', J. Aust. Math. Soc. 108 (2020), 120-144.

[47] J.-M. Souriau, Structure des systèmes dynamiques (Dunod, Paris, 1970).

[48] M. Spera, 'A survey on the differential and symplectic geometry of linking numbers', Milan J. Math. 74 (2006), 139-197.

[49] M. Spera, 'Moment map and gauge geometric aspects of the Schrödinger and Pauli equations', Int. J. Geom. Methods Mod. Phys. 13(4) (2016), 1630004.

[50] J. N. Tavares, 'Chen integrals, generalized loops and loop calculus', Int. J. Mod. Phys. A 9 (1994), 4511-4548.

[51] F. Warner, Foundations of Differentiable Manifolds and Lie Groups, Graduate Texts in Mathematics, 94 (Springer, Berlin, 1983).

[52] G. J. Zuckerman, 'Action principles and global geometry', Proceedings of the Conference on Mathematical Aspects of String Theory, San Diego, CA, 21 July-2 August 1986 (ed. S. T. Yau) (World Scientific, Singapore, 1987), 259-284.

ANTONIO MICHELE MITI, Dipartimento di Matematica e Fisica, Università Cattolica del Sacro Cuore,

Via dei Musei 41, 25121 Brescia, Italy

and

Department of Mathematics, KU Leuven,

Celestijnenlaan 200B Box 2400, 3001 Leuven, Belgium

e-mail: antoniomichele.miti@unicatt.it

MAURO SPERA, Dipartimento di Matematica e Fisica,

Università Cattolica del Sacro Cuore,

Via dei Musei 41, 25121 Brescia, Italy

e-mail: mauro.spera@unicatt.it 